\title{
REGIONAL INHIBITION OF SYMPATHETIC TONE DURING SHIVERING AND INDUCED MUSCLE CONTRACTION*†
}

\author{
D. W. DuCharmeł and L. Beck \\ Department of Pharmacology, University of Michigan, Ann Arbor, Michigan
}

(Accepted 13 January 1966)

\begin{abstract}
Summary-Adrenergic vasoconstriction evoked by the stimulation of the decentralized lumbar sympathetic chain can be inhibited during muscle contraction induced by shivering, sciatic nerve stimulation, or activation of the viscero-somatic reflex. Receptors which initiate the inhibition are thought to be present in skeletal muscle and activated by stretch. The specific site at which the inhibition occurs has not been determined but is known to be at a locus in the efferent adrenergic pathway peripheral to the spinal cord.
\end{abstract}

\section{INTRODUCTION}

IT HAS generally been accepted by most investigators that activity in the sympathetic nervous system is controlled primarily by inhibitory influences acting at the medullary vasomotor centres. However, Lim et al. (1938) have suggested that sympathetic activity can be controlled at the level of the spinal cord as well. More recently, Gebrer et al. (1963), BECK (1963), GeBBeR and BtCK (1963) showed that sympathetic activity can also be modulated by inhibitory influences acting at the sympathetic chain ganglia or perhaps even at a more distant locus. The peripheral inhibition reported by Gebber and Beck was shown to emanate from centres located in the central nervous system and to originate at least in part from receptors located in the conventional buffer zones of the carotid sinus and aortic arch. Other work dealing with the mechanism of serotonin dilatation led us to believe that regional reflex inhibition of adrenergic tone may result from stimulation of receptors located in the periphery (GeBber, 1964; GeBber and BeCK, 1964). From a hypothetical viewpoint, such a system of regional inhibition would be most useful in exercise. Inhibition of adrenergic tone only in the exercising region would result in a shunting of blood to the exercising area. The shunting would be greater if adrenergic vasoconstrictor effect to other areas were simultaneously increased.

To test this hypothesis the effect of sympathetic nerve stimulation upon the vasculature of the perfused hindlimb of the dog was examined in the presence and absence of skeletal muscle activity.

* Presented in part at the 1964 rall meetings of the American Society for Pharmacology and Experimental Therapeutics in Lawrence, Kansas. (Du ChaRME and BECK, 1964).

† Supported by grants HE-03946 and He-07511 from United States Public Health Service.

\& Present address: Pharmacology Research Laboratories, The Upjohn Company, Kalamazoo, Michigan. 


\section{METHODS}

Mongrel dogs, unselected as to sex and weighing from 11 to $20 \mathrm{~kg}$, were used in this investigation. All animals were anesthetized with $30 \mathrm{mg} / \mathrm{kg}$ of sodium pentobarbital intravenously, and received $5 \mathrm{mg} / \mathrm{kg}$ sodium heparin to prevent blood coagulation. The hindquarters were prepared for perfusion by the method described in detail by BECK (1961). The abdominal aorta was exposed using a retroperitoneal approach through a longitudinal incision in the left flank and ligated opposite the body of the third lumbar vertebrae. Blood was led from a cannula placed in the proximal portion of the aorta and delivered by means of a Sigmamotor pump to a cannula placed in the distal portion of the aorta. The blood flow to the extremities was arbitrarily adjusted at the beginning of the experiment so that the perfusion pressure approximately equalled the systemic blood pressure. After initial adjustment of the pump, blood flow to the hindquarters was kept constant throughout the experiment.

In those preparations in which only a single hindlimb was perfused, the aorta was doubly cannulated and a T-tube interposed in the tubing connecting the proximal and distal aortic cannulae. Blood led off from the T-tube by a Sigmamotor pump was delivered to a cannula inserted into only one iliac artery. In the preparations in which each of the two hindlimbs was perfused independently, separate Sigmamotor pumps were employed to supply blood to the left and right iliac arteries.

Systemic blood pressure was recorded on smoked k'mograph paper by means of a double arm mercury manometer attached to a side arm of the cannula inserted into the proximal aorta. The perfusion pressure of the hindquarters was recorded by means of a single arm mercury manometer attached to a side arm of the cannula inserted into the distal aorta. Drugs were administered intra-arterially by injecting them through a piece of rubber tubing to the distal cannula. Drugs administered intravenously were injected into the jugular vein.

The lumbar sympathetic trunks were exposed in the mid-lumbar area. Harvard bipolar shielded electrodes were used for stimulation. When it was desirable to interrupt adrenergic discharge to the extremities, the sympathetic trunks were sectioned or repeatedly crushed in the same segment as, but just cephalad to, the stimulating electrode. In some experiments reflex contraction of skeletal muscle of the ipsilateral hindlimb was initiated by stimulation of afferent fibers in the sympathetic trunks. In such experiments the sympathetic chain was divided in the interganglionic segment immediately cephalad to the segment upon which the electrode was placed. The sciatic nerve was approached through the gluteal muscles. A section 1-2 cm in length was exposed for stimulation. American Electronic Laboratory Stimulators were used in all cases for stimulating the nerves.

Skeletal muscle activity was induced in the perfused hindlimb by one of the following three methods: (1) ice packs were placed on shaven areas of the dog to precipitate shivering; (2) the sciatic nerve was electrically stimulated; (3) a reflex contraction of the hindleg musculature was induced by stimulation of visceral afferent fibres in the sympathetic chain.

\section{RESULTS}

\section{Effect of skeletal muscle activity on sympathetic tone}

Skeletal muscle activity consistently and selectively depressed neurogenically induced vasoconstriction in the perfused hindlimb of the dog. Representative experiments illustrating this phenomenon are shown in Fig. 1. The record at the top of Fig. 1 was taken 
In the dog shown in the lower record of Fig. 1, skeletal muscle activity was initiated by stimulating the intact sciatic nerve supplying the perfused limb instead of by induction of shivering. The first panel shows the constrictor responses obtained by efferent stimulation of the decentralized lumbar sympathetic chain and by the intra-arterial injection of norepinephrine. The same procedures were then carried out during sciatic nerve stimulation. As shown in the second panel, the response to sympathetic nerve stimulation was reduced much out of proportion to the norepinephrine-induced response, the relative reductions being 60 and 16 per cent, respectively. Control responses were again obtained after sciatic nerve stimulation was discontinued. The neurogenic and norepinephrine responses were now indistinguishable from those obtained previous to sciatic nerve stimulation. It is worthwhile noting that the non-neurogenic vascular tone remaining in the limb after acute lumbar sympathetic transection (which is equivalent to acute sympathectomy) was reduced by one-third during sciatic nerve stimulation. This reduction, as well as the smaller reduction in the norepinephrine response, demonstrates that vascular tone can be non-specifically decreased during motor activity.

The effect of motor activity induced by low voltage stimulation of the sciatic nerve upon adrenergic responses induced by lumbar sympathetic chain stimulation and by intra-arterial injection of norepinephrine was tested in 13 experiments. The neurogenically induced constriction was reduced by an average of $44.5 \pm 1.29$ per cent. The norepinephrine induced constriction was reduced by an average of $12 \cdot 7 \pm 1 \cdot 13$ per cent. The difference is very significant $(P<0.001)$.

When shivering is arrested there is a selective increase in the constrictor response produced by sympathetic stimulation. Such an increase is shown in the upper record of Fig. 2 in which shivering in the extremities was arrested by cord transection in the upper lumbar area. Following cord section, the neurogenic response produced by stimulation of the lumbar sympathetic trunk at $1 \mathrm{c} / \mathrm{s}$ increased to 182 per cent of the response obtained during shivering. At $0.5 \mathrm{c} / \mathrm{s}$ the neurogenic response was increased to 260 per cent of the control response. In contrast norepinephrine-induced constriction was fractionally decreased from the control response obtained during shivering.

A similar selective increase in the response to sympathetic stimulation was seen when shivering was prevented in 5 dogs by spinal anesthesia. The neurogenic constriction, produced by efferent stimulation of the acutely sectioned lumbar sympathetic, returned to $129 \cdot 6 \pm 1 \cdot 36$ per cent of the value present before induction of shivering. The response to norepinephrine rose to $125.8 \pm 3.2$ per cent of the norepinephrine constriction present before shivering. In 8 other dogs in which shivering was arrested by administration of the neuromuscular blocking agent decamethonium, the neurogenic response returned to $107.9 \pm 1.59$ per cent of the pre-shivering response. In these dogs, the norepinephrine response returned to 109.9 per cent of the pre-shivering response produced by intraarterial injection of norepinephrine.

The selective depression of neurogenically evoked constriction produced by motor stimulation of the sciatic nerve also disappears abruptly upon discontinuation of the sciatic nerve stimulation as was shown in the lower record of Fig. 1.

Stimulation of the intact sympathetic trunk often evokes a reflex contraction of the hindlimb musculature. 'Ihis reflex is called the viscero-somatic reflex. 'I he reflex contraction persists when the lumbar chain is sectioned in the segment cephalad to the stimulating electrode, but it is abolished by crushing or sectioning the chain in the same segment immediately cephalad to the stimulating electrode. This makes it possible to test 

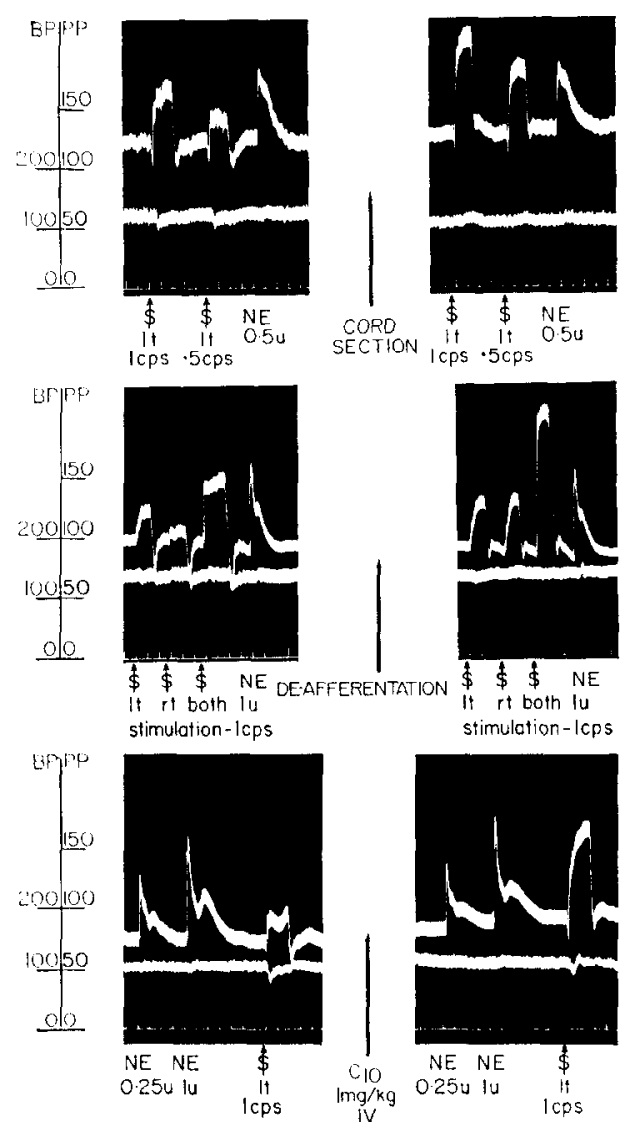

FIG. 2. (Upper record.) Effect of abolishing shivering by spinal cord section at L1 on preganglionic lumbar sympathetic nerve stimulation and intra-arterial injection of norepinephrine. Upper tracing is perfusion pressure of left hindleg only. Symbols are the same as in Fig. 1. (Middle record.) Effect of removing viscero-somatic reflex twitch by de-afferentation on preganglionic lumbar sympathetic nerve stimulation. Upper tracing is the perfusion pressure to the hindquarters. Symbols are the same as in Fig. 1. (Lower record.) Effect of removing the viscero-somatic reflex twitch by decamethonium on the response to preganglionic lumbar sympathetic nerve stimulation. Upper tracing in the perfusion pressure to the left hind leg only. $\mathrm{rt}=$ Stimulation of right lumbar sympathetic trunk; other symbols are the same as in

Fig. 1.

the influence of the viscero-somatic reflex upon neurogenic tone elicited in the extremity by preganglionic lumbar sympathetic stimulation.

The middle record of Fig. 2 was taken from a dog in which the two lumbar sympathetic chains had been previously sectioned in the segment ahead of the stimulating electrode, thus leaving one ganglion with rami intact cephalad to the electrode. Stimulation thus activated both afferent fibers passing out over rami ahead of the electrode and efferent fibcrs passing from the chain over rami below the electrode. The responses to stimulation under these conditions for the left and right lumbar chains, separately and together, are shown in the first panel of the middle record. The control response obtained upon intraarterial injection of norepinephrine is also illustrated. 
The responses to stimulation and to norepinephrine injection after sectioning of the sympathetic chain in the same segment as the stimulating electrode, but immediately proximal to it, are shown in the right hand panel of the middle record in Fig. 2. The response to left lumbar sympathetic stimulation was increased 1.6 times by section, the response to right lumbar sympathetic chain stimulation was increased $5 \cdot 5$ times by section, and the response to combined stimulation of the two chains was increased $2 \cdot 2$ times by section. The constriction produced by the intra-arterial injection of norepinephrine remained unchanged.

Abolition of the viscero-somatic reflex caused the greatest potentiation of the neurogenic constriction on the side in which the reflex skeletal muscle contraction had appeared to be greater, but no objective evaluation of the strength of contraction has been made.

The record shown at the bottom of Fig. 2 illustrates a similar selective increase in the lumbar sympathetic response when the viscero-somatic reflex is prevented by treatment with the neuromuscular blocking agent rather than by deafferentation proximal to the electrode. The record is taken from a dog in which only the left hind extremity was perfused. When reflex skeletal muscle contraction was prevented by administration of 1 $\mathrm{mg} / \mathrm{kg}$ of intravenous decamethonium, the vascular constriction produced by lumbar sympathetic nerve stimulation was increased threefold without change in the response to intra-arterially injected norepinephrine, showing that abolition of muscle contraction is the essential factor leading to the potentiation.

\section{DISCUSSION}

The finding that the vasoconstrictor response evoked by electrical excitation of the lumbar sympathetic nerves progressively declined as the intensity of spontaneous or induced shivering increased cannot be attributed to a simple physiologic or independent antagonism between a metabolic dilator substance released from contracting skeletal muscle and a neurogenic constrictor released from adrenergic nerves. If sufficient dilator substances were being released from skeletal muscle during shivering to antagonize the constriction produced by neurogenically released norepinephrine, then enough dilator metabolites should also be released to antagonize an equivalent constriction produced by the intra-arterial injection of norepinephrine into the same perfused bed. Since it was repeatedly observed and statistically confirmed that a marked reduction in the neurogenic response occurred during shivering with only a minor concomitant decrease in the response to injected norepinephrine, an explanation based on simple physiologic antagonism can be ruled out. (To avoid misunderstanding the authors wish to point out that it is not their contention that shivering or other forms of induced skeletal muscle activity cannot reduce the constriction produced by injected norepinephrine. If muscle activity is intense enough in the dog there is always a reduction in the response to any injected constrictor agent. The point being emphasized is that during milder states of muscle activity there is a selective reduction of the neurogenic response).

Neither can the selective reduction in the neurogenic response be attributed to some indirect consequence of cooling such as failure of ganglionic or nerve transmission, prevention of adrenergic amine release, exhaustion of the adrenergic depot or the like. The neurogenic response, which is reduced when shivering is induced, is immediately restored by administration of the neuromuscular blocking agent, decamethonium, before any 
detectable change in body temperature occurs. If shivering is arrested by deepening the pentobarbital anesthesias an increase in the response to electrical stimulation also occurs. Consequently, it must be assumed that shivering selectively inhibits adrenergic vasoconstriction induced by electrical stimulation of the decentralized preganglionic lumbar sympathetic chain.

It is almost universally agreed that blood flow in the extremities is increased as body temperature is elevated and decreased as body temperature is lowered (SHEPHERD, 1963). In addition, it has been shown that the blood flow alterations which occur in the extremities upon varying the body temperature can be attributed primarily to changes in resistance of the cutaneous vasculature (SHEPHERD, 1963). Although no studies on skin blood flow, per se, appear to have been done during shivering, our results obtained before and after skinning the perfused limb indicate that the skin blood flow is very small compared to muscle blood flow. Removal of the skin during perfusion at constant blood flow alters the perfusion pressure only by a very small amount. In no case has it increased the perfusion pressure by more than 25 per cent, and usually the increases have been much less. Since it therefore appears that most of the blood flow in the extremity is distributed to the muscle bed, it follows that increases in vascular tone of the skin vessels induced by lumbar sympathetic stimulation would have a relatively small effect upon the perfusion pressure to the whole extremity even if constriction of the skin vessels were marked. Our experimental results agree with this conclusion, since in our experiments skinning of the hindlimb during constant blood flow (which would simulate complete closure of the skin blood vessels during sympathetic stimulation) had rather small effect upon the perfusion pressure and no apparent effect upon the neurogenic vasoconstrictor response produced by lumbar sympathetic stimulation. Therefore, it can be inferred that the changes in resistance observed in our experiments reflect events transpiring primarily in the skeletal muscle vascular bed.

From a theoretical point of view it might be supposed that shivering could stimulate a central inhibitory system whose efferent fibers were distributed to the peripheral sympathetic system. Such an inhibitory system has been recently described (BECK, 1963; GeBBER and Beck, 1963). The system has been shown to inhibit tone induced in the hind extremity by efferent stimulation of the divided lumbar sympathetic. It seems unlikely, however, that shivering activates centrally the peripherally distributed inhibitory system. Induction of skeletal muscle contraction by other means also causes a selective depression in the lumbar sympathetic response. Thus reflex contraction of the hind limb muscles produced by afferent sympathetic stimulation or muscle contraction produced by low voltage sciatic nerve stimulation also preferentially reduces nerve induced constriction more than norepinephrine induced constriction. It has been further shown that inhibition of adrenergic activity persists in both of the latter cases when the spinal cord is transected and disappears when neuromuscular blockade is produced. It was also shown that neuromuscular blockade abolishes shivering and simultaneously removes the inhibition to the adrenergic system. But neuromuscular blockade does not remove the peripherally distributed inhibition reported by BECK (1963), and GEBBER and BECK (1963). Consequently, it can be inferred that the two types of inhibition are effected in different ways.

The most plausible explanation for the motor inhibition is that contraction of skeletal muscle stimulates receptors within the contracting muscle which in some manner inhibit the adrenergic vasoconstrictor effect. Such a mechanism has been previously postulated by MitCHell et al. (1963), who assumed that the afferents stimulated during skeletal 
muscle contraction discharged into the central nervous system to inhibit adrenergic vaso constriction. But in our studies, unlike those of Mitchell et al., spontaneous tone was intentionally removed at the beginning of the experiment by transection of the lumbar sympathetics. Tone was then periodically replaced by stimulation of the efferent lumbar sympathetic. Consequently, an afferent system which suppresses adrenergic activity by inhibition in the medulla could exert no influence in our experiments upon adrenergic tone being induced by peripheral sympathetic nerve stimulation. Accordingly, the inhibition of adrenergic activity observed in the present investigation must have occurred in the periphery between the point of electrode placement on the lumbar sympathetic and the responding vessels in the limb. Because the adrenergic nerves do not re-enter the spinal cord before peripheral distribution, the site of inhibition can be neither in the medulla nor the spinal cord and must therefore be extraspinal.

While the site at which the inhibition occurs is thus localized to a point outside of the spinal cord, it cannot be inferred from the cxperiments so far citcd that the hypothetical afferent fiber does not enter the cord to cmerge again over the ventral root before entering the lumbar sympathetic chain. This possibility was tested by determining the effect of spinal anesthesia on the inhibition of adrenergic vasoconstriction observed during muscle activity evoked by sciatic nerve stimulation. Table 1 shows that sciatic nerve stimulation

TABLE, 1 INHIBIION OF INDUCED ADRENERGIC VASOCONSIRICTION BY SCIATIC STIMULATION BLFORE AND ATTER SPINAL. ANESTHESIA

\begin{tabular}{cccccc}
\hline Control* & $\begin{array}{c}\text { Before spinal } \\
\text { Sciatic } \\
\text { stim.* }\end{array}$ & $\begin{array}{c}\% \\
\text { reduction }\end{array}$ & Control* $^{*}$ & $\begin{array}{c}\text { After spinal } \\
\text { Sciatic } \\
\text { stim.* }\end{array}$ & $\begin{array}{c}\% \\
\text { reduction }\end{array}$ \\
\hline 1.01 & 0.53 & 48 & 1.24 & 0.8 & 35 \\
1.7 & 0.33 & 81 & 1.42 & 0.82 & 42 \\
1.33 & 0.56 & 58 & 1.21 & 0.52 & 57 \\
0.95 & 0.59 & 38 & 0.88 & 0.4 & 55 \\
\hline Average & & 56.2 & & & 47.2 \\
\hline
\end{tabular}

*Figures represent the ratio of sympathetic stimulation response/norepinephrine response.

produced an average reduction of 56 per cent in the neurogenic constrictor response before spinal anesthesia; and an average reduction of 47 per cent after spinal anesthesia. Presumptively spinal anesthesia was complete in these animals, since sufficient local anesthetic was administered to abolish not only the patellar reflex but also spontaneous respiration.

From these results it must be presumed that the inhibition of adrenergic vasoconstriction was occurring primarily outside of the spinal cord. But whether the inhibition of neurogenic constriction is effected by a local reflex involving the regional ganglia, by an axon reflex in the periphery, or by the elaboration of a humoral substance which simply prevents release of norepinephrine from local adrenergic endings cannot be asccrtaincd from these experiments.

\section{REFERENCES}

BECK, L. (1961). Active reflex dilatation in the innervated perfused hindleg of the dog. Am. J. Physiol. 201: $123-128$.

BECK, L. (1963). A new concept in autonomic control. Periphcral inhibition and disinhibition. Univ. Mich. Med. Bull. 29: 292-296. 
DUCHARME, D. W. and BeCK, L. (1964). Regional inhibition of sympathetic tone during shivering. Pharmacologist 6: 184.

Gebber, G. L. (1964). Doctoral Dissertation: Reftex Inhibition of Adrenergic Activity at a Peripheral Locus. The University of Michigan pp. 1-217.

Gebrer, G. L. and Beck, L. (1963). Reflex nature of ganglionic inhibition. Pharmacologist 5: 244.

GeBBeR, G. L. and BECK, L. (1964). Local reflex actions of serotonin. Pharmacologist 6: 184.

Gebber, G. L., Kayaalp, S. O., DuCharme, D. W. and Beck, L. (1963.) Evidence for ganglionic inhibition of sympathetic tone. Univ. Mich. Med. Bull. 29: 175-181.

LIM, R. K. S., WANG, S. C., and YI C. L. (1938). On the question of a myelencephalic sympathetic centre VII. Clin. J. Physiol. 13: 61-77.

Mitchell, J. H., Remensnyder, J. P. and Sarnoff, S. J. (1963.) Vasomotor responses in a resting musculocutaneous area during muscular activity. Am J. Physiol. 205: 37-40.

Shipherd, J. T. (1963). Physiology of the Circulation in Human Limbs in Health and Disease. W. O. Saunders, Philadelphia and London p. 416. 